BMJ Open

Diabetes

Research

\& Care

\title{
Prevalence of diabetes and cardiovascular risk factors in middle- class urban participants in India
}

\begin{abstract}
Arvind Gupta, ${ }^{1}$ Rajeev Gupta, ${ }^{2}$ Krishna Kumar Sharma, ${ }^{3}$ Sailesh Lodha, ${ }^{2}$ Vijay Achari, ${ }^{4}$ Arthur J Asirvatham, ${ }^{5}$ Anil Bhansali, ${ }^{6}$ Balkishan Gupta, ${ }^{7}$ Sunil Gupta, ${ }^{8}$ Mallikarjuna V Jali, ${ }^{9}$ Tulika G Mahanta, ${ }^{10}$ Anuj Maheshwari, ${ }^{11}$ Banshi Saboo, ${ }^{12}$ Jitendra Singh, ${ }^{13}$ Prakash C Deedwania ${ }^{14}$
\end{abstract}

To cite: Gupta A, Gupta R, Sharma KK, et al. Prevalence of diabetes and cardiovascular risk factors in middle-class urban participants in India. BMJ Open Diabetes Research and Care 2014;2:e000048. doi:10.1136/bmjdrc-2014000048

Received 28 July 2014 Revised 7 October 2014 Accepted 29 October 2014

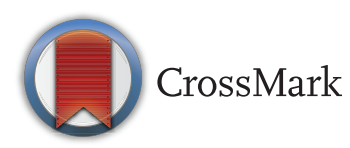

For numbered affiliations see end of article.

Correspondence to Dr Rajeev Gupta; rajeevgg@gmail.com

\section{ABSTRACT}

Objectives: To determine the prevalence of diabetes and awareness, treatment and control of cardiovascular risk factors in population-based participants in India.

Methods: A study was conducted in 11 cities in different regions of India using cluster sampling. Participants were evaluated for demographic, biophysical, and biochemical risk factors. 6198 participants were recruited, and in 5359 participants (86.4\%, men $55 \%$ ), details of diabetes (known or fasting glucose $>126 \mathrm{mg} /$ $\mathrm{dL}$ ), hypertension (known or blood pressure $\geq 140$ / $\geq 90 \mathrm{~mm} \mathrm{Hg}$ ), hypercholesterolemia (cholesterol $\geq 200 \mathrm{mg} / \mathrm{dL}$ ), low high-density lipoprotein (HDL) cholesterol (men $<40$, women $<50 \mathrm{mg} / \mathrm{dL}$ ), hypertriglyceridemia (>150 mg/dL), and smoking/ tobacco use were available. Details of awareness, treatment, and control of hypertension and hypercholesterolemia were also obtained.

Results: The age-adjusted prevalence (\%) of diabetes was $15.7(95 \% \mathrm{Cl} 14.8$ to 16.6 ; men 16.7 , women 14.4$)$ and that of impaired fasting glucose was 17.8 (16.8 to 18.7; men 17.7, women 18.0). In participants with diabetes, $27.6 \%$ were undiagnosed, drug treatment was in $54.1 \%$ and control (fasting glucose $\leq 130 \mathrm{mg} / \mathrm{dL}$ ) in $39.6 \%$. Among participants with diabetes versus those without, prevalence of hypertension was 73.1 (67.2 to 75.0 ) vs 26.5 (25.2 to 27.8), hypercholesterolemia 41.4 (38.3 to 44.5 ) vs 14.7 (13.7 to 15.7 ), hypertriglyceridemia 71.0 (68.1 to 73.8 ) vs 30.2 (28.8 to 31.5), low HDL cholesterol 78.5 (75.9 to 80.1) vs 37.1 (35.7 to 38.5), and smoking/smokeless tobacco use in 26.6 (23.8 to 29.4) vs 14.4 (13.4 to $15.4 ; p<0.001$ ). Awareness, treatment, and control, respectively, of hypertension were $79.9 \%, 48.7 \%$, and $40.7 \%$ and those of hypercholesterolemia were $61.0 \%, 19.1 \%$, and $45.9 \%$, respectively.

Conclusions: In the urban Indian middle class, more than a quarter of patients with diabetes are undiagnosed and the status of control is low. Cardiovascular risk factors-hypertension, hypercholesterolemia, low HDL cholesterol, hypertriglyceridemia, and smoking/smokeless tobacco use-are highly prevalent. There is low awareness, treatment, and control of hypertension and hypercholesterolemia in patients with diabetes.

\section{Key messages}

More than a quarter of patients with diabetes in the Indian urban middle class are undiagnosed.

- In patients with diabetes, cardiovascular risk factors-hypertension, hypercholesterolemia, low high-density lipoprotein cholesterol, and hypertriglyceridemia-are highly prevalent.

- The status of awareness, treatment, and control of hypertension and hypercholesterolemia is low.

\section{INTRODUCTION}

Diabetes is endemic in India. ${ }^{1-3}$ The International Diabetes Federation has estimated that India currently has more than 65 million people with type 2 diabetes and the numbers are poised to double in the next 20 years. ${ }^{1}$ It has been reported that the prevalence of diabetes among urban participants in India is among the highest in the world and comparable to the high prevalence countries of West Asia and the Pacific. ${ }^{3}{ }^{4}$ Cardiovascular diseases (coronary heart disease, stroke, peripheral arterial disease) are the major causes of morbidity and mortality in type 2 diabetes. It has been reported that $60-80 \%$ of patients with diabetes die of cardiovascular events. ${ }^{5}{ }^{6}$ Reasons for the increased risk include the high prevalence of major cardiovascular risk factors (hypertension, lipid abnormalities, and smoking) as well as factors specific to diabetes (hyperglycemia, diabetic dyslipidemia, and oxidation-related and glycation-related vascular injury). ${ }^{7}$

Control of cardiovascular risk factors in diabetes can prevent or delay cardiovascular events. Studies have reported that therapies directed toward the control of blood pressure (BP) and low density lipoprotein (LDL) cholesterol can significantly decrease macrovascular events in diabetes. ${ }^{8} 9$ In India, a high 
prevalence of metabolic cardiovascular risk factors has been reported among clinic-based patients with diabetes. ${ }^{10}$ Only a few population-based studies in India have determined the prevalence of various cardiovascular risk factors in patients with diabetes. ${ }^{11-13}$ No study has evaluated the status of awareness, treatment and control of hypertension and hypercholesterolemia in population-based patients with diabetes . Therefore, we performed this study to determine the prevalence of diabetes, impaired fasting glycemia (IFG) and various cardiovascular risk factors in populationbased patients in India with diabetes. We also studied the awareness, treatment, and control of hypertension and hypercholesterolemia, two of the most important cardiovascular risk factors.

\section{METHODS}

A multisite study to identify the prevalence of cardiovascular risk factors and their sociodemographic determinants was conducted among middle-class urban participants in India. ${ }^{14-17}$ The rationale for the study has been reported. ${ }^{18}$ The protocol was approved by the institutional ethics committee of the national coordinating center. Written informed consent was obtained from each participant. The study case report form was developed according to the guidelines of the WHO. ${ }^{19}$

\section{Regions and investigators}

We planned the study to identify the prevalence of cardiometabolic risk factors and their determinants in middle-class urban participants in India. ${ }^{18}$ Briefly, medium-sized cities were identified in each of the large states of India and investigators with track record of research in cardiovascular or diabetes epidemiology were invited. A steering committee and investigators meeting was organized at the initiation of the study where the study protocol was discussed and developed. The meeting was followed by training in the salient features of the questionnaire and techniques of examination and evaluation to ensure uniformity in recruitment and data collection. Eleven investigators in 11 cities finally performed the survey. ${ }^{14}$ These cities are distributed in all the geographic regions of the country —northern (Jammu, Chandigarh), western (Bikaner, Ahmedabad), southern (Belgaum, Madurai), central (Nagpur, Jaipur), and eastern (Lucknow, Patna, Dibrugarh).

\section{Sampling}

The study data were collected in the years 2006-2010 at various locations. Simple cluster sampling was performed at each site. A middle-class location was identified at each city by the investigator. The middle-class location is based on municipal classification and is derived from the cost of land, type of housing, public facilities (roads, sanitation, water supply, electricity, gas supply, etc), and educational and medical facilities as reported earlier. ${ }^{14}$ A sample size of about 250 men and 250 women $(n=500)$ at each site is considered adequate by the WHO to identify a $20 \%$ difference in the mean level of biophysical and biochemical risk factors. ${ }^{19}$ We invited 800-1000 participants in each location to ensure participation of at least 500 participants at each site, estimating a response of $70 \%$ as observed in previous studies. ${ }^{20}$ At each site, a uniform protocol of recruitment was followed. A locality within the urban area of the city was identified on ad hoc basis by each investigator, houses enumerated, number of participants $\geq 20$ years living in each house determined and all these individuals were invited to a local community center or healthcare facility (clinic, dispensary) for examination and blood investigations. This procedure ensured that the study was representative even if the survey was prematurely abandoned. ${ }^{14}$ The surveys were preceded by meetings with community leaders to ensure good participation. Participants were invited in the fasting state to a community center or medical center within each locality, either twice or thrice a week, depending on the investigator's schedule.

\section{Measurements}

The study case report form was filled by the research worker after details were inquired from the participant. Apart from demographic history, details of socioeconomic status based on educational status and years of formal education, type of family, any major previous illnesses, history of known hypertension, diabetes, lipid abnormalities, and cardiovascular disease were inquired. Smoking details were inquired for the type of smoking or tobacco use, number of cigarettes or bidis smoked, and years of smoking or smokeless tobacco use. Intake of alcohol was assessed as drink per week. Details of other diet and physical activity were inquired using focused questions. ${ }^{14}$ All the equipments for measurements of height, weight, waist and hip size, and BP were similar at the centers to ensure uniformity. Height and weight were measured using a stadiometer and calibrated weighing machines, respectively, and waist and hip circumference was measured using the WHO guidelines. ${ }^{19}$ Sitting BP was measured after at least 5 min rest using standardized instruments. Three readings were obtained and were averaged for the data analysis. A fasting blood sample was obtained from all individuals after at least $8-10 \mathrm{~h}$ fast. The blood samples were obtained at community centers by technicians from an accredited national laboratory-Thyrocare Technologies Ltd, Mumbai, India (http://www.thyrocare.com). Blood glucose was measured at the local biochemistry facility of these laboratories. Blood for cholesterol, cholesterol lipoproteins, and triglyceride estimation was transported under ambient temperature to the national referral laboratory at Mumbai. All the blood samples were analyzed at a single laboratory and a uniform protocol was 
used for measurements. ${ }^{16}$ Cholesterol, high-density lipoprotein (HDL) cholesterol, and triglyceride levels were measured using enzyme-based assays with internal and external quality control. LDL cholesterol was calculated using Friedwald's formula. ${ }^{16}$

\section{Diagnostic criteria}

Smokers included participants who smoked cigarettes, bidis, or other smoked forms of tobacco daily, while past smokers were participants who had smoked for at least 1 year and had stopped more than a year ago. Use of other forms of tobacco (oral, nasal, etc) was classified as smokeless tobacco. Individuals with greater than moderate physical activity (30 min of work-related or leisuretime physical activity, $\geq 5$ times a week) were classified as moderately active. Those with a high dietary intake of visible fat ( $\geq 30 \mathrm{~g}$ visible fat intake/day) and $\leq 2$ dishes of fruits or green vegetables/day were classified as having an unhealthy diet. Hypertension was diagnosed when systolic BP was $>140 \mathrm{~mm} \mathrm{Hg}$ and/or diastolic BP was $\geq 90 \mathrm{~mm} \mathrm{Hg}$ or a person was a known hypertensive. ${ }^{15}$ Hypercholesterolemia was defined by total cholesterol $\geq 200 \mathrm{mg} / \mathrm{dL}$, low HDL cholesterol by levels $<40 \mathrm{mg} / \mathrm{dL}$ in men and $<50 \mathrm{mg} / \mathrm{dL}$ in women, and hypertriglyceridemia as $\geq 150 \mathrm{mg} / \mathrm{dL} .{ }^{16}$ Diabetes was diagnosed when either a participant was diagnosed by a physician or the fasting blood glucose was $\geq 126 \mathrm{mg} / \mathrm{dL}$. Participants with fasting glucose $100-125 \mathrm{mg} / \mathrm{dL}$ were diagnosed as those with IFG. Known diabetes by history, those on any drug therapy as being on treatment, and control was defined by fasting glucose $\leq 130 \mathrm{mg} / \mathrm{dL}$ according to American Diabetes Association/European Association of Study of Diabetes criteria. ${ }^{21}$ The prevalence of various cardiovascular risk factors was determined in participants with and without diabetes. The status of awareness, treatment, and control of hypertension and hypercholesterolemia was also determined using previous definitions. ${ }^{15} 16$ Hypertension control was defined when systolic BP was $<140 \mathrm{~mm} \mathrm{Hg}$ and diastolic BP was $<90 \mathrm{~mm} \mathrm{Hg}$. Controlled hypercholesterolemia was defined by the presence of total cholesterol $<200 \mathrm{mg} / \mathrm{dL} .^{21}$

\section{Statistical analyses}

All the data were entered into the SPSS database (V.10.0, SPSS Inc, Chicago, Illinois, USA). More than $90 \%$ of the data for various variables were available, and in about $85 \%$ of participants the data for all the variables were available. Categorical variables are reported as per cent and $95 \%$ CIs. The prevalence of diabetes and IFG in various age groups is reported. Age-related trends were examined by the Mantel-Haenszel $\chi^{2}$ test. Age adjustment was performed using the direct method with the 2001 Indian census population as the standard. Prevalence $(\%)$ of various risk factors in participants with diabetes and without diabetes is reported and significance of differences evaluated using $\chi^{2}$ test. $p$ Values $<0.05$ are considered significant.

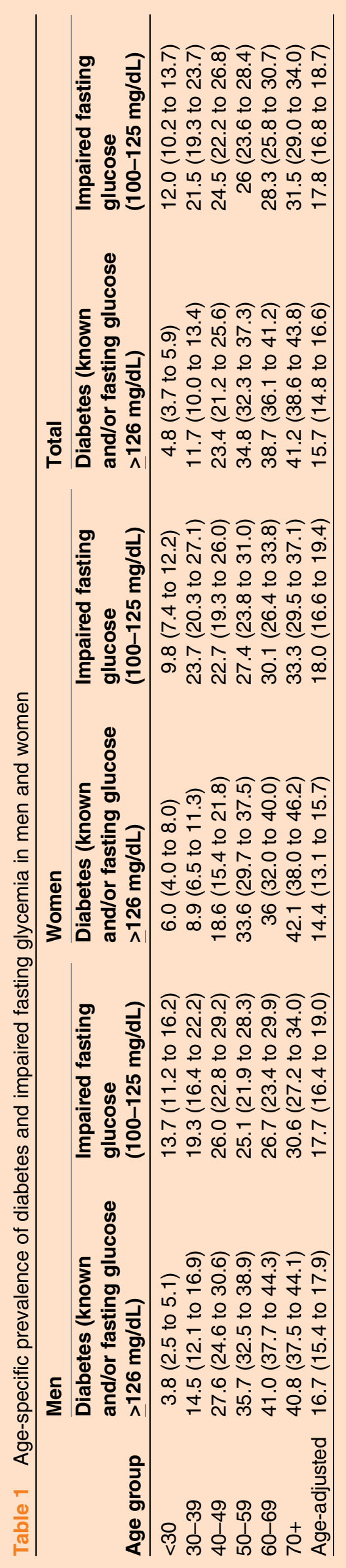




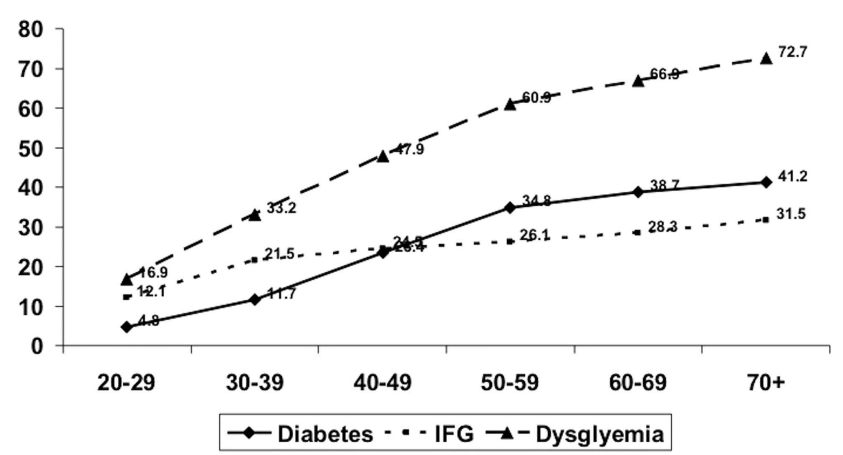

Figure 1 Age-specific prevalence of diabetes, impaired fasting glycemia (IFG), and any dysglycemia.

\section{RESULTS}

The study was performed at 11 cities located in all geographic regions of India as reported earlier. ${ }^{14}$ In total, 6198 participants (men 3426, women 2772) of the targeted 9900 participants were evaluated (response 62\%). Recruitment at individual sites and data for social and demographic characteristics in men and women have been reported. ${ }^{14}$ Among 5359 participants (men 54.8\%, women $45.2 \%$ ), details of diabetes and various cardiovascular risk factors were available, and therefore these data have been used in the present study.

The age-specific prevalence $(\%)$ of diabetes and IFG and $95 \%$ CI is shown in table 1 . There is a significant age-associated increase in the prevalence of diabetes in men and women $(\mathrm{p}<0.001)$. The age-adjusted prevalence of diabetes was $15.7 \%$ (CI $14.8 \%$ to $16.6 \%$ ); in men, it was $16.7 \%$ (CI $15.4 \%$ to $17.9 \%$ ), and in women it was $14.4 \%(13.1 \%$ to $15.7 \%)$. The age-adjusted prevalence of IFG was $17.8 \%$ (CI $16.8 \%$ to $18.7 \%$ ); in men, it was $17.7 \%$ (CI $16.4 \%$ to $19.0 \%$ ), and in women it was $18.0 \%$ (CI $16.6 \%$ to $19.4 \%$ ). The prevalence of IFG was greater than that of diabetes in the younger age groups and lower in the older age groups (figure 1). The prevalence of diabetes and IFG equalized at a younger age in men as compared with women (table 1). The prevalence of any dysglycemia (diabetes or IFG) also shows an age-associated increase and is present in more than $60 \%$ among older participants (figure 1).
Of the study participants with diabetes, presence of known disease was in $72.4 \%$ (CI $69.6 \%$ to $75.2 \%$ ) and was greater in older participants (table 2). Almost a quarter were undiagnosed (27.6\%, CI $24.8 \%$ to $30.4 \%$ ). Of the total patients with diabetes, drug treatment was present in $54.1 \%$ (CI $51.0 \%$ to $57.2 \%$ ), and in those with previous diagnosis it was present in $74.7 \%$ (CI $71.8 \%$ to $77.6 \%)$. The status of diabetes control was defined using fasting glucose $<130 \mathrm{mg} / \mathrm{dL}$. $^{21}$ Among all patients with diabetes, control was present in $39.6 \%$ (CI $36.5 \%$ to $42.7 \%$ ), and in those with previously diagnosed diabetes it was present in $48.2 \%$ (CI $44.5 \%$ to $51.9 \%$ ).

The age-specific prevalence of various risk factors in participants with diabetes is shown in table 3. Among participants with diabetes versus those without, age-adjusted prevalence of hypertension was in $73.1 \%$ (CI $67.2 \%$ to $75.0 \%$ ) vs $26.5 \%$ (CI $25.2 \%$ to $27.8 \%$ ), hypercholesterolemia in $41.4 \%$ (CI $38.3 \%$ to $44.5 \%$ ) vs $14.7 \%$ (CI $13.7 \%$ to $15.7 \%$ ), low HDL cholesterol in $78.5 \%$ (CI $75.9 \%$ to $80.1 \%$ ) vs $37.1 \%$ (CI $35.7 \%$ to $38.5 \%$ ), hypertriglyceridemia in $71.0 \%$ (CI $68.1 \%$ to $73.8 \%$ ) vs $30.2 \%$ (CI $28.8 \%$ to $31.5 \%)$, and smoking and/or smokeless tobacco use in $26.6 \%$ (CI $23.8 \%$ to $29.4 \%$ ) vs $14.4 \%$ (CI $13.4 \%$ to $15.4 \%) ;(p<0.01$; figure 2$)$. The prevalence of smoking was in $18.2 \%$ vs $8.9 \%$ while that of smokeless tobacco use was present in $13.5 \%$ vs $7.9 \%$ ( $\mathrm{p}<0.01)$. In known patients with diabetes versus others, hypertension awareness ( $79.9 \%$ vs $43.2 \%)$, treatment $(48.7 \%$ vs $31.3 \%)$, and control $(40.7 \%$ vs $20.6 \%)$ as well as hypercholesterolemia awareness $(61.0 \%$ vs $12.0 \%)$, treatment $(19.1 \%$ vs $5.0 \%)$, and control (total cholesterol $<200 \mathrm{mg} / \mathrm{dL}, 45.9 \%$ vs $6.8 \%$; LDL cholesterol $<100 \mathrm{mg} / \mathrm{dL}, 66.5 \%$ vs $8.6 \%)$ were significantly greater $(\mathrm{p}<0.01$; figure 3$)$.

\section{DISCUSSION}

The International Diabetes Federation has reported that the prevalence of diabetes in adults in India is $7.1 \%$. The prevalence in urban areas is $9 \% .^{1}$ This study shows a greater prevalence among the middle-class urban Indians. The study also shows that participants with diabetes have a high prevalence of major cardiovascular risk factors-hypertension, hypercholesterolemia, low

Table 2 Prevalence $(\%, 95 \% \mathrm{Cl})$ of diabetes and status of diagnosis, treatment, and control in various age groups

\begin{tabular}{|c|c|c|c|c|c|c|}
\hline Age group & Numbers & $\begin{array}{l}\text { Diabetes (known } \\
\text { and/or fasting } \\
\text { glucose } \\
\geq 126 \mathrm{mg} / \mathrm{dL} \text { ) }\end{array}$ & Known diabetes & Undiagnosed & Treatment & $\begin{array}{l}\text { Control, fasting } \\
\text { glucose } \\
\leq 130 \mathrm{mg} / \mathrm{dL}\end{array}$ \\
\hline$<30$ & 417 & 4.7 (2.7 to 6.7$)$ & 30.0 (27.3 to 32.7$)$ & 70.0 (64.8 to 75.2$)$ & $15.0(12.6$ to 17.5$)$ & 35.0 (30.4 to 39.6$)$ \\
\hline $30-39$ & 976 & $11.7(9.7$ to 13.7$)$ & 58.8 (55.9 to 61.7$)$ & 41.2 (35.6 to 46.8 ) & 37.7 (34.4 to 40.9$)$ & 34.2 (31.2 to 37.2$)$ \\
\hline $40-49$ & 1445 & $23.4(21.2$ to 25.6$)$ & 75.7 (73.2 to 78.2 ) & 24.3 (19.4 to 29.2$)$ & $53.8(50.4$ to 57.1$)$ & 38.1 (35.6 to 40.6$)$ \\
\hline $50-59$ & 1313 & 34.8 (32.2 to 37.4$)$ & 82.7 (80.5 to 84.9 ) & 17.2 (12.9 to 21.5$)$ & 63.0 (59.7 to 66.2$)$ & 43.3 (40.6 to 46.0 ) \\
\hline $60-69$ & 859 & 38.7 (35.4 to 41.9$)$ & 85.2 (83.1 to 87.3 ) & 14.7 (10.7 to 18.7$)$ & 70.9 (67.8 to 73.9$)$ & 42.0 (38.7 to 45.3$)$ \\
\hline $70+$ & 349 & 41.2 (36.0 to 46.4$)$ & 81.9 (79.6 to 84.2$)$ & 22.8 (18.0 to 22.6$)$ & 67.4 (64.2 to 70.5$)$ & 46.5 (41.3 to 51.7$)$ \\
\hline Age-adjusted & 5359 & $15.7(14.7$ to 16.7$)$ & 72.4 (69.6 to 75.2 ) & 27.6 (24.8 to 30.4$)$ & 54.1 (51.0 to 57.2$)$ & 39.6 (36.5 to 42.7$)$ \\
\hline
\end{tabular}




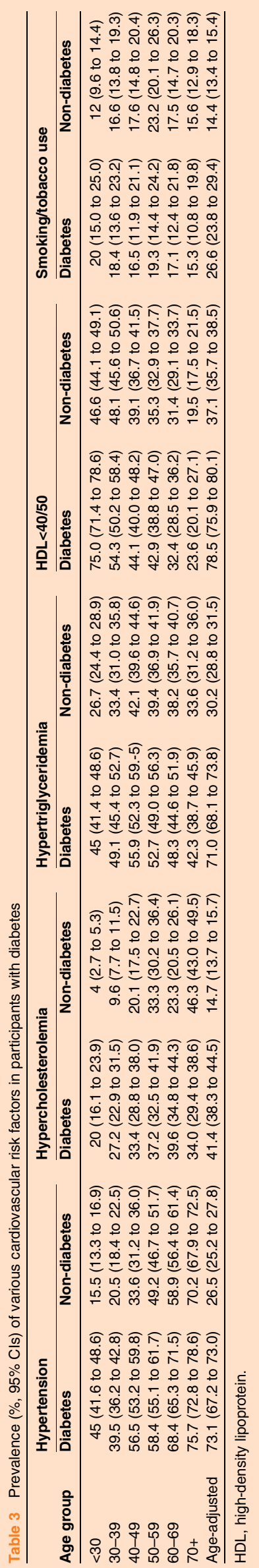

HDL cholesterol, and high triglycerides. The status of diabetes control as well as treatment and control of two important cardiovascular risk factors (hypertension and hypercholesterolemia) is low.

Previous studies in India have reported a greater diabetes prevalence in urban adults as compared with rural adults. ${ }^{2-4}$ Recent studies have reported urban diabetes prevalence rates of $8-20 \%$ and rural diabetes prevalence rates of $5-15 \% .^{2}$ There are only a few multisite studies of diabetes prevalence in India. Using criteria similar to that used in this study, the Indian Industrial Population Surveillance Study evaluated the prevalence of diabetes at seven industrial sites in the country and reported a diabetes prevalence of $8 \% .^{22}$ The Indian Women's Health Study evaluated diabetes in middle-aged women, 3570 years, in different rural and urban locations of the country and reported diabetes in 2.2\% rural and 9.3\% urban women. ${ }^{23}$ The India Migration Study reported diabetes in $13.5 \%$ urban, $14.3 \%$ migrant, and $6.2 \%$ rural participants. ${ }^{24}$ Multisite studies such as DESI, ${ }^{25}$ PODIS, ${ }^{26}$ and INDIAB ${ }^{27}$ used fasting as well as $2 \mathrm{~h}$ glucose estimation for diagnosis of diabetes, and therefore the results are not comparable to those of this study. Regional studies have reported a greater prevalence of diabetes in Southern India as compared with the north, east, and central India. ${ }^{24}$ This study reports a prevalence of $15.7 \%$, which is similar to that reported in recent studies from India. We have not presented data on the regional differences in diabetes prevalence due to the small sample sizes (500-1000) at different locations. However, we have earlier shown that regional differences in diabetes prevalence are related more to the Social Development Index ${ }^{28}$ of the cities and not to the geography; cities with lower poverty indices and better social development indices have a greater prevalence of diabetes and other cardiometabolic risk factors. ${ }^{29}$ These results are similar to those of studies from China ${ }^{30}$ and other middle-income countries. ${ }^{31}$ Larger and more comprehensive studies are required to identify regional differences in diabetes and to evaluate the causes of these differences. ${ }^{32}$

This study also shows a high prevalence of major cardiovascular risk factors (hypertension, hypercholesterolemia, low HDL cholesterol, hypertriglyceridemia, and smoking/smokeless tobacco use) in participants with diabetes (figure 2). This finding is similar to those from studies from other parts of the world. ${ }^{6} 7$ The greater prevalence of smoking as well as smokeless tobacco use in participants with diabetes is an important finding and is similar to those of previous Indian studies. ${ }^{33}$ Another important finding is the low prevalence of these risk factors in the population without diabetes and suggests that diabetes is a major driver of cardiometabolic risks in India. ${ }^{7}$ A high prevalence of undiagnosed diabetes in younger participants ( $<40$ years) is also important and highlights the importance of surveillance and screening among the younger populations for early diagnosis of diabetes in India. This study also shows that diabetes is associated with multiple cardiovascular risk factors and 
Figure 2 Age-adjusted prevalence of various cardiovascular risk factors in diabetes and others. The prevalence of all risk factors is significantly greater in diabetes $(p<0.01)$. HDL, high density lipoprotein cholesterol; values of cholesterol and triglycerides are in $\mathrm{mg} / \mathrm{dL}$.

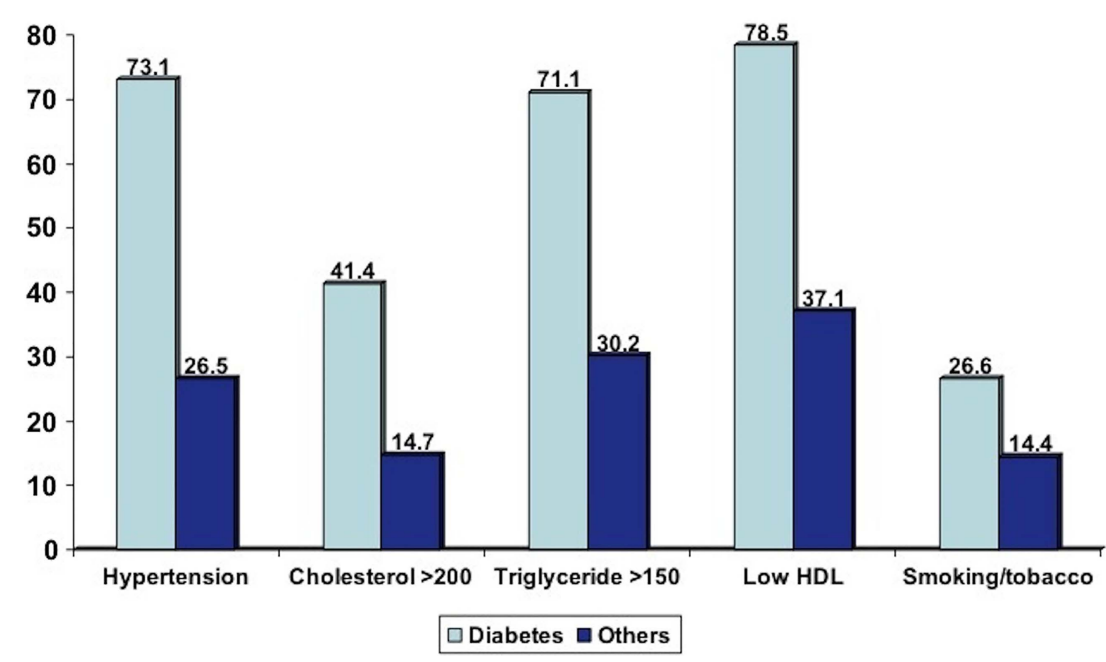

its prevention and elimination can have important consequences for cardiovascular disease prevention.

The prevalence of known diabetes in this study is more than $70 \%$ of all individuals with diabetes and is more than those shown by previous reports from India. ${ }^{11-13}$ We studied the urban middle-class participants and found that the greater prevalence of known diabetes in this population was indicative of greater health literacy. ${ }^{14}$ We have previously reported higher awareness of hypertension in the present study participants $^{15}$, this is similar to high awareness of diabetes in the present study. However, only half of the patients with diabetes are controlled to the target of fasting glucose $<130 \mathrm{mg} / \mathrm{dL}$. This is similar to previous reports from India. ${ }^{11}$ The American Diabetes Association and the European Association for Study of Diabetes recommend three markers for the assessment of diabetes control (blood fasting glucose, postprandial glucose, or glycated hemoglobin (HbAlc)). ${ }^{21}$ We defined control using fasting glucose levels only and did not measure blood HbA1c. This is an important study limitation.

The low status of awareness, treatment, and control of cardiovascular risk factors among participants with diabetes is also an important finding in this study. Only a few international studies have evaluated the status of cardiovascular risk factor control in patients with diabetes. Gakidou $e t a l^{34}$ compared the management of diabetes and associated cardiovascular risk factors in seven countries (Colombia, England, Iran, Mexico, Scotland, Thailand, and the USA). This study reported that a substantial proportion of individuals with diabetes remain undiagnosed and untreated in various developed and developing countries and ranged from $24 \%$ in Scotland and the USA to $62 \%$ in Thailand. The proportion of individuals with diabetes reaching treatment targets for blood glucose, systolic BP, and cholesterol ranged from $1 \%$ in Mexico to $12 \%$ in the USA. ${ }^{34}$ Low control of diabetes and hypertension has also been reported in a study in India. ${ }^{35}$ Our study also shows a low status of control of hypertension and hypercholesterolemia. More studies are required to confirm these findings. The greater awareness, treatment, and control of cardiovascular risk factors in participants with known diabetes observed in this study (figure 3) is similar to that reported in studies in the USA. ${ }^{36}$

Other limitations of the study are biases introduced because of sampling, non-representation of the Indian
Figure 3 Status of awareness, treatment, and control of hypertension and hypercholesterolemia (total cholesterol $\geq 200 \mathrm{mg} / \mathrm{dL}$ ) in study participants with known diabetes and others.

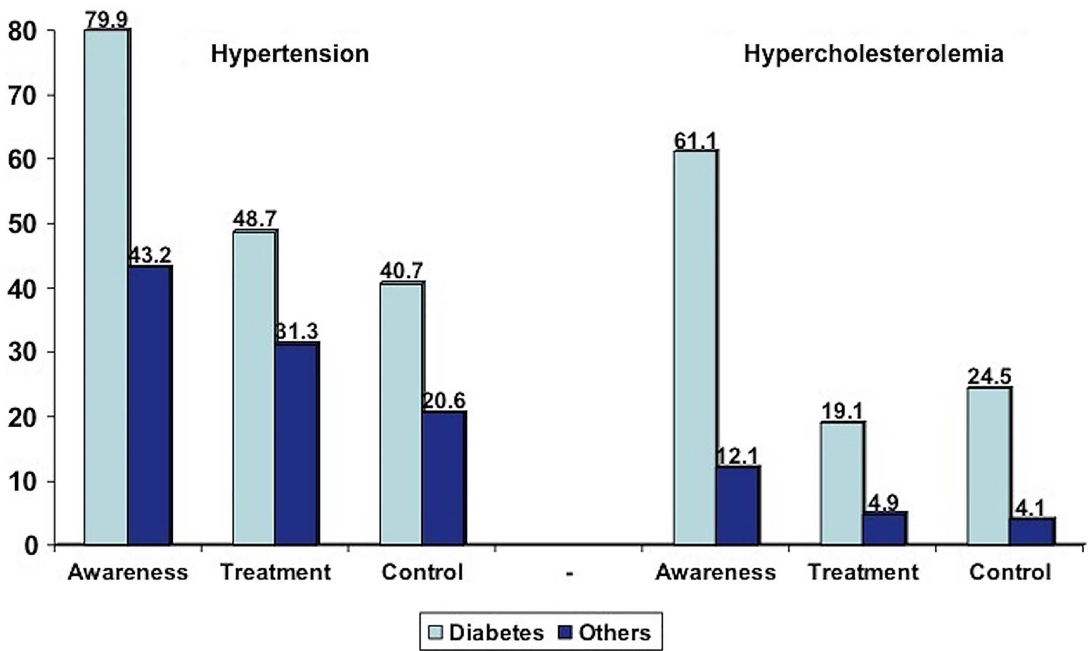


population, inclusion of only urban participants, low response rates, measurement techniques, and failure to correct for regression dilution. However, many of the limitations are inherent in a cross-sectional epidemiological study and the data are therefore subject to similar biases. ${ }^{14}$ Urban locations are hotbeds of the cardiovascular disease epidemic in India, ${ }^{4}$ and this study is therefore important. Moreover, similar methodology has been used in previous Indian studies and the present data are similarly representative. ${ }^{18}$ The low response rate in the study (62\%) is also a matter of concern and it is possible that those excluded were either more or less healthy as compared with the study participants; however, these response rates are similar to those of other population-based studies in India and elsewhere ${ }^{19}$ and are within acceptable limits. ${ }^{37}$ Finally, there are multiple determinants of awareness, treatment, and control of cardiovascular risk factors in patients with diabetes. We have not analyzed the 'causes of the causes' or the societal factors ${ }^{38}$ that lead to greater cardiovascular risk and better awareness of risk factors in study participants. On the other hand, the strengths of the study include a nationwide scope and study of multiple risk factors.

In conclusion, our nationwide study shows that the prevalence of diabetes among the urban middle-class participants in India is greater than that reported by the International Diabetes Federation. ${ }^{1}$ There is a low status of treatment and control. This study also shows a high prevalence of multiple cardiovascular risk factors among participants with diabetes. The low status of control of hypertension and hypercholesterolemia in participants with known diabetes is a cause for concern. Suitable strategies for improvement of risk factor management and control should be developed in India to prevent premature cardiovascular disease in diabetes.

\section{Author affiliations}

${ }^{1}$ Department of Diabetes, Jaipur Diabetes Research Centre, Jaipur, Rajasthan, India

${ }^{2}$ Departments of Medicine and Endocrinology, Fortis Escorts Hospital, Jaipur, Rajasthan, India

${ }^{3}$ Department of Pharmacy, SMS Medical College, Rajasthan University of Health Sciences, Jaipur, Rajasthan, India

${ }^{4}$ Department of Medicine, Patna Medical College, Patna, Bihar, India

${ }^{5}$ Department of Medicine, Government Medical College, Madurai, Tamil Nadu, India

${ }^{6}$ Department of Endocrinology, Postgraduate Institute of Medical Education and Research, Chandigarh, India

${ }^{7}$ Department of Medicine, SP Medical College, Bikaner, Rajasthan, India

${ }^{8}$ Department of Diabetes, Diabetes Care and Research Centre, Nagpur, Maharashtra, India

${ }^{9}$ Department of Medicine, JN Medical College, Belgaum, Karnataka, India

${ }^{10}$ Department of Community Medicine, Assam Medical College, Dibrugarh, Assam, India

${ }^{11}$ Department of Medicine, BBD College of Dental Sciences, Lucknow, Uttar Pradesh, India

${ }^{12}$ Department of Diabetes, DiaCare and Research, Ahmadabad, Gujarat, India

${ }^{13}$ Department of Medicine, Government Medical College, Jammu, Jammu and Kashmir, India

${ }^{14}$ Department of Cardiology, University of California San Francisco VA Medical Center, Fresno, California, USA
Funding The study was financially supported by the South Asian Society for Atherosclerosis and Thrombosis, Bangalore (India) and Minneapolis (USA) grant number Nil of 2006.

Contributors RG, PCD, and AG designed the study and developed the protocol; they were also involved in obtaining funding, investigator training, and supervised the whole study. $A G$ and $R G$ jointly wrote the first draft and subsequent drafts of the article. VA, AJA, AB, BG, SG, MVJ, TGM, AM, BS, and JS were the site investigators and supervised the conduct of the study locally. They were involved in data collection and provided inputs for the article and also critically reviewed the whole article and provided suggestions. $A G, R G$, and $K K S$ were involved in the study supervision, data management, and statistical analyses. All authors have read the manuscript and agree to its contents.

\section{Competing interests None.}

\section{Patient consent Obtained.}

Ethics approval Institutional Ethics Committee, Fortis Escorts Hospital, Jaipur, India.

Provenance and peer review Not commissioned; externally peer reviewed.

Data sharing statement No additional data are available.

Open Access This is an Open Access article distributed in accordance with the Creative Commons Attribution Non Commercial (CC BY-NC 4.0) license, which permits others to distribute, remix, adapt, build upon this work noncommercially, and license their derivative works on different terms, provided the original work is properly cited and the use is non-commercial. See: http:// creativecommons.org/licenses/by-nc/4.0/

\section{REFERENCES}

1. International Diabetes Federation. 6th edn. IDF diabetes Atlas, 2013 http://www.idf.org/diabetesatlas

2. Anjana RM, Ali MK, Pradeepa R, et al. The need for obtaining accurate nationwide estimates of diabetes prevalence in India: rationale for a national study on diabetes. Indian $J$ Med Res 2011;1133:369-80.

3. Ramachandran A, Ma RC, Snehalatha C. Diabetes in Asia. Lancet 2010;375:408-18

4. Gupta R, Misra A. Type-2 diabetes in India: regional disparities. $\mathrm{Br} \mathrm{J}$ Diabetes Vasc Dis 2007;7:12-16.

5. Huxley R, Barzi F, Woodward M. Excess risk of fatal coronary heart disease associated with diabetes in men and women: meta-analysis of 37 prospective studies. BMJ 2006;332:73-8.

6. Holman RR, Sourij H, Califf RM. Cardiovascular outcome trials of glucose lowering drugs or strategies in type 2 diabetes. Lancet 2014;383:2008-17.

7. Volkova NB, Deedwania PC. The metabolic syndrome and its effects on cardiovascular risks. In: Fonseca V, ed. Clinical diabetes: translating research into practice. Philadelphia. Saunders, 2006:64-77

8. Bangalore S, Kumar S, Lobach I, et al. Blood pressure targets in subjects with type 2 diabetes mellitus/impaired fasting glucose: observations from traditional and Bayesian random-effects meta-analyses of randomized trials. Circulation 2011;123:2799-810.

9. De Vries FM, Denig P, Pouwels KB, et al. Primary prevention of major cardiovascular and cerebrovascular events with statins in diabetic patients: a meta-analysis. Drugs 2012;72:2365-73.

10. Sridhar GR, Putcha V, Lakshmi G. Time trends in the prevalence of diabetes mellitus: ten year analysis from southern India (1994-2004) on 19,072 subjects with diabetes. J Assoc Physicians India 2010;58:290-4.

11. Mohan V, Venkatraman JV, Pradeepa R. Epidemiology of cardiovascular disease in type 2 diabetes the Indian scenario. J Diabetes Sci Technol 2010;4:158-70.

12. Ramachandran A, Mary S, Yamuna A et al. High prevalence of diabetes and cardiovascular risk factors associated with urbanization in India. Diabetes Care 2008;31:893-8.

13. Misra A, Pandey RM, Sharma R. Non communicable diseases (diabetes, obesity and hyperlipidemia) in urban slums. Natl Med $J$ India 2002;15:242-4.

14. Gupta R, Deedwania PC, Sharma KK, et al. Association of education, occupation and socioeconomic status with cardiovascular risk factors in Asian Indians: a cross-sectional study. PLoS ONE 2012;7:e044098. 
15. Gupta R, Deedwania PC, Achari V, et al. Normotension, prehypertension and hypertension in Asian Indians: prevalence, determinants, awareness, treatment and control. Am J Hypertens 2013;26:83-94.

16. Guptha S, Gupta R, Deedwania PC, et al. Cholesterol lipoproteins, triglycerides and prevalence of dyslipidemias among urban Asian Indian subjects: a cross sectional study. Indian Heart $J$ 2014;66:280-8.

17. Deedwania PC, Gupta R, Sharma KK, et al. High prevalence of metabolic syndrome among urban subjects in India: a multisite study. Diabetes Metab Syndr 2014;8:156-61.

18. Gupta R, Guptha S, Sharma KK, et al. Regional variations in cardiovascular risk factors in India: India Heart Watch. World $\mathrm{J}$ Cardiol 2012;4:112-20.

19. Luepkar RV, Evans A, McKeigue P, et al. Cardiovascular survey methods. 3rd edn. Geneva: World Health Organization, 2002.

20. Gupta R, Gupta VP, Sarna M, et al. Prevalence of coronary heart disease and risk factors in an urban Indian population: Jaipur Heart Watch-2. Indian Heart J 2002;54:59-66.

21. Inzucchi SE, Diamant M, Ferranini E, et al. Management of hyperglycemia in type 2 diabetes: a patient centered approach Position statement of the American Diabetes Association (ADA) and the European Association for the Study of Diabetes (EASD). Diabetes Care 2012;35:1364-79.

22. Reddy KS, Prabhakaran D, Chaturvedi V, et al. Methods for establishing a surveillance system for cardiovascular diseases in Indian industrial populations. Bull World Health Organ 2006;84:461-9.

23. Pandey RM, Gupta R, Misra A, et al. Determinants of urban-rural differences in cardiovascular risk factors in middle-aged women in India: a cross-sectional study. Int J Cardiol 2013;163:157-62.

24. Ebrahim S, Kinra S, Bowen L, et al.; Indian Migration Study Group. The effect of rural-to-urban migration on obesity and diabetes in India: a cross sectional study. PLoS Med 2010;7:e10000268.

25. Ramachandran A, Snehalatha C, Kapur A, et al. Diabetes Epidemiology Study Group in India (DESI). High prevalence of diabetes and impaired glucose tolerance in India: National Urban Diabetes Survey. Diabetologia 2001;44:1094-101.

26. Sadikot SM, Nigam A, Das S, et al.; DiabetesIndia. The burden of diabetes and impaired glucose tolerance in India using the WHO 1999 criteria: prevalence of diabetes in India study (PODIS). Diabetes Res Clin Pract 2004;66:301-7.
27. Anjana RM, Pradeepa R, Deepa M, et al:; ICMR-INDIAB Collaborative Study Group. Prevalence of diabetes and prediabetes (impaired fasting glucose and/or impaired glucose tolerance) in urban and rural India: phase I results of the Indian Council of Medical Research-INdia DIABetes (ICMR-INDIAB) study. Diabetologia 2011;54:3022-7.

28. Banerjee K. Social development index 2010. In: Mohanty M, ed. India Social Development Report 2010. New Delhi: Oxford University Press, 2011:259-93.

29. Gupta R, Deedwania PC, Sharma KK, et al. Urban social development index as determinant of cardiovascular risk in India. Abstract. Circulation 2012;125(Suppl):A0083.

30. Attard SM, Herring AH, Mayer-Davis EJ, et al. Multilevel examination of diabetes in modernizing China: what elements of urbanization are most associated with diabetes? Diabetologia 2012;55:3182-92.

31. Rydin Y, Bleahu A, Davies M, et al. Shaping cities for health: complexity and the planning of urban environments in the 21st century. Lancet 2012;379:2079-108.

32. Corsi DJ, Subramanian SV. Association between socioeconomic status and self-reported diabetes in India: a cross sectional multilevel analysis. BMJ Open 2012;2:e000895.

33. Thankappan KR, Mini GK, Daivadanam M, et al. Smoking cessation among diabetes patients: results of a pilot randomized trial. $B M C$ Public Health 2013;13:47.

34. Gakidou E, Mallinger L, Abbott-Klafter J, et al. Management of diabetes and associated cardiovascular risk factors in seven countries: a comparison of data from national health examination surveys. Bull World Health Organ 2011;89:172-83.

35. Joshi SR, Das AK, Vijay V, et al. Challenges in diabetes care in India: sheer numbers, lack of awareness and inadequate control. J Assoc Physicians India 2008;56:443-50.

36. Brown TM, Tanner RM, Carson AP, et al. Awareness, treatment and control of LDL cholesterol are lower among US adults with undiagnosed diabetes versus diagnosed diabetes. Diabetes Care 2013;36:2734-40.

37. Johnson TP, Wislar JS. Response rates and non-response errors in surveys. JAMA 2012;307:1805-6.

38. Marmot M, Friel S, Bell R, et al.; Commission on Social Determinants of Health. Closing the gap in a generation: health equity through action on the social determinants of health. Lancet 2008;372: $1661-9$. 